\title{
TRANSFERENCIAS CONDICIONADAS E IGUALDAD DE GÉNERO: ¿BLANCOS, NEGROS O GRISES??
}

\author{
BLACKS, WHITES OR GREYS? \\ CONDITIONAL TRANSFERS AND GENDER EQUALITY
}

\author{
Juliana Martínez Franzoni* \\ Koen Voorend**
}

RESUMEN

¿En qué medida las transferencias condicionadas (TMC), programas "estrella" de la nueva generación de la política social latinoamericana, inhiben o promueven mayores grados de igualdad de género? Usando un instrumento teórico-empírico novedoso, y a partir de fuentes primarias y secundarias, nuestro análisis compara tres países con regímenes de bienestar contrastantes: Chile, Costa Rica y El Salvador. Mostramos que, aunque en términos de la igualdad de género, los programas TMC se basan en supuestos que en principio exacerban la histórica división del trabajo entre hombres y mujeres, también pueden transformarla.

PALABRAS CLAVES: BIENESTAR SOCIAL * POLÍTICA SOCIAL * GÉNERO * EQUIDAD * POBREZA

\section{ABSTRACT}

To what extent do conditional cash transfer (CCT) programs, the "star" programs of the new generation of Latin American public policy, promote or inhibit greater degrees of gender equality? Using a novel theoretical-empirical tool for the study of gender equality, and based on primary and secondary information, our analysis

1 Instituto de Investigaciones Sociales, Universidad de Costa Rica. Este artículo es parte de una investigación más amplia que contó con el apoyo de la Fundación Carolina y del Observatorio de la Desigualdad del Centro de Estudios Latinoamericanos de la Universidad de Miami. Agradecemos además la asistencia de Nayla Carbajal y de Héctor Solano, así como valiosos comentarios de Merike Blofield y Carolina Ávalos. Los errores y las omisiones son nuestros.

\footnotetext{
* Instituto de Investigaciones Sociales. Universidad de Costa Rica. martinezfranzoni@gmail.com

** Instituto de Investigaciones Sociales. Universidad de Costa Rica.

koenvoorend@gmail.com
} 
compares three countries with contrasting welfare regimes, Chile, Costa Rica and El Salvador. In terms of gender equality, although CCT programs are based on assumptions that exacerbate the historical division of labour between women and men, they can also debilitate it.

\section{KEY WORDS: SOCIAL WELFARE * SOCIAL POLICY * GENDER * EQUALITY * POVERTY}

\section{INTRODUCCIÓN}

El papel de las mujeres $y$ de las relaciones de género se ha instalado en el debate público sobre la política social en América Latina, involucrando voces $y$ actores que trascienden a las organizaciones promotoras de la equidad de género. El detonante ha sido los programas de transferencias condicionadas, mediante los cuales, aunque con diferencias en su diseño, los gobiernos entregan dinero a cambio de que las madres envíen a sus niños y niñas a la escuela $y$ a los centros de salud. Aunque la importancia relativa de estos programas en el gasto público social es pequeña en comparación con otros sectores de política social, su importancia radica en ser epicentro de una nueva generación de política social que se articula de maneras diversas con las políticas preexistentes. Discusiones relativas a si estos programas realmente permiten superar la pobreza, cómo y entre quiénes, aparecen de la mano de si es "apropiado", "justo" o "necesario" que movilicen el tiempo y la energía de millones de mujeres madres sin que ellas sean también sujetas y no solo instrumentos de la política. Así, e independientemente de si son efectivos o no y para qué, estos programas, que a octubre del 2008 existían en 15 de 18 países de América Latina, han tenido la virtud de haber puesto en el tapete la importancia del género como mediador de la relación entre Estado y sociedad ${ }^{2}$.

Aunque las evaluaciones abundan tanto como los programas, hasta ahora no han sido analizados a la luz de los entornos, laborales

Molyneux, Maxine. "Change and Continuity in Social Protection in Latin America: Mothers at the Service of the State?". Documento de trabajo nro. 1. Ginebra: UNRISD, 2007. $y$ sociales, en los cuales estos programas se insertan. Además, consideramos como una gran limitación, el que las valoraciones sobre los efectos que tienen en las relaciones de género, carezcan de una noción explícitamente multidimensional de la equidad. Sabemos que la histórica división del trabajo entre hombres proveedores de ingresos y mujeres cuidadoras, conlleva una jerarquía mediante la cual se crean y recrean relaciones de subordinación de los hombres hacia las mujeres. A la vez, sabemos que la subordinación económica no siempre tiene lugar conjuntamente con otras formas de subordinación, como la sexual o la relativa al uso del tiempo. Se requiere entonces, "desagregar" la igualdad de género en más de una dimensión, y determinar cómo las transferencias condicionadas moldean unas u otras.

Para llenar estos vacíos, este estudio aborda las consecuencias que para la equidad de género tienen tres programas de transferencias monetarias condicionadas (en adelante, TMC) en el marco de constelaciones de relaciones entre mercados laborales, políticas sociales y familias - es decir, en el marco de los regímenes de bienestar-. ¿Se está, desde esta política social, promoviendo cambios en las relaciones de género tradicionales? ¿ $\mathrm{O}$, por el contrario, se está poniendo la división sexual del trabajo tradicional al servicio del Estado?, o tal vez ambas cosas a la vez y, si fuera así, ¿por qué?

\section{LAS TMC EN LOS REGÍMENES DE BIENESTAR}

En América Latina hay diferentes constelaciones de relaciones entre mercados laborales, política social y familias, es decir, regímenes de bienestar. Estas constelaciones dan lugar a 
prácticas de asignación de recursos que permitan a las personas acceder, mejor o peor, a bienes y servicios. Martínez Franzoni identifica tres principales regímenes de bienestar según los grados de mercantilización de la fuerza de trabajo, nacional o trasnacional; de desmercantilización, es decir de autonomía entre los ingresos y el bienestar de las personas; $y$ de desfamiliarización, referente en que el bienestar deja de estar sujeto a la disponibilidad de trabajo femenino no remunerado (Martínez Franzoni, 2008).

\section{CUADRO 1 \\ CHILE SOLIDARIO (CHILE)}

El Sistema de Protección y Promoción Social Chile Solidario (en adelante "Chile Solidario"), se creó en el 2002 bajo la administración de Ricardo Lagos (2000-06). Se dirige a familias en extrema pobreza, a las cuales el programa da acceso preferencial a la red nacional, regional y local de transferencias y servicios sociales, de acuerdo a características y necesidades específicas. Las transferencias varían entre US\$ 5,9 y 19,8 al mes. De acuerdo al gobierno de Chile, en noviembre del 2008, el programa alcanzaba a 332995 familias. Según CEPAL (2007), en el 2005 se cubría a un $6,45 \%$ del total de la población mediante la movilización de un $0,1 \%$ del PIB (CEPAL, 2007). Chile Solidario es ejecutado por los municipios y comienza con el Programa Puente. Este combina apoyo psicosocial y una transferencia condicionada durante 24 meses y se ejecuta con un/a acompañante o Apoyo Familiar. El segundo componente es el de subsidios monetarios, entre los cuales se encuentran: el Subsidio Único Familiar, para todos los niños y niñas menores de 18 años; la Pensión Asistencial de Vejez, para todos los mayores de 65 años; la Pensión Asistencial de Invalidez para quienes corresponda; $y$ el Subsidio de Agua Potable, para cubrir el $100 \%$ de la cuenta hasta 15 metros cúbicos de consumo mensual. El tercer componente conlleva la prioridad en el acceso a programas sociales correspondientes a las áreas de salud, educación, trabajo, vivienda, justicia, entre otras; sectores que a su vez, y para ello, asumen compromisos con el Ministerio de Planificación (MIDEPLAN).

Fuente: Gobierno de Chile (2006) y Chile Solidario, sitio web: <http://www.chilesolidario.gov.cl> [consultado el 07/07/2009].

Teniendo la cautela necesaria para interpretar tipologías en tanto simplificaciones $y$ exageraciones de ciertos aspectos de la realidad, ella identificó tres principales regímenes, según el Estado promueva principalmente un manejo mercantil, estatal o familiar de los riesgos sociales. Así lo documentan 21 indicadores estadísticamente significativos arrojados por el análisis de conglomerados para 18 países.

\section{CUADRO 2 \\ AVANCEMOS (COSTA RICA)}

Comenzó a implementarse en el 2006 bajo la administración Arias (2006-2010). Se basa en una integración del sistema de prestación de becas otorgado por el Fondo Nacional de Becas (FONABE), ubicado en el Ministerio de Educación desde la Administración Pacheco (20022006), y las transferencias dirigidas al combate a la pobreza desde el Instituto Mixto de Ayuda Social (IMAS). En octubre del 2008, Avancemos llegaba a 130586 estudiantes (Secretaría Técnica del Programa AVANCEMOS, 2008). El programa combina la entrega de una transferencia monetaria a jóvenes pertenecientes a familias de escasos ingresos que estén matriculados en el colegio secundario, a cambio de su permanencia en el sistema educativo. Inicialmente la administración de los recursos podía hacerse desde la institución de combate a la pobreza, IMAS, o desde el FONABE, en el Ministerio de Educación: el primero contempla a la madre como administradora del dinero, mientras que el segundo trasfiere los recursos directamente a los/as jóvenes. Desde el 2009, sin embargo, todas las transferencias se canalizan a través del IMAS. Desafortunadamente, CEPAL (2007) carece de datos comparativos sobre el peso del programa Avancemos en el PIB, aunque sí reporta datos relativos al programa piloto que le precedió, Superémonos, y que representaba un $0,02 \%$ del PIB en el 2005.

Fuente: Contraloría General de la República (2008) y Secretaría Técnica del Programa AVANCEMOS (2008).

Chile, Costa Rica y El Salvador son países emblemáticos de estos tres regímenes de bienestar. Entre estos varía, primero, la efectividad de los mercados laborales para absorber y remunerar la fuerza de trabajo sin que una gran proporción de la población deba forzosamente emigrar. En Chile y Costa Rica esta capacidad es en términos relativos alta, mientras que en El Salvador es baja. Por ejemplo, en Chile la población asalariada alcanza el $74 \%$ de la población y de la independiente calificada solo el $15 \%$, en comparación a El Salvador adonde las cifras son del $47 \%$ y $30 \%$, respectivamente. A la vez, las remesas alcanzan menos del $1 \%$ en Chile y el $2 \%$ en Costa Rica, pero superan el 
15\% en El Salvador. Segundo, entre estos países varía la presencia relativa de hogares nucleares tradicionales, con hombre proveedor y mujer cuidadora. Mientras en Chile, un 54\% de los hogares están organizados de esta manera, en Costa Rica desciende al 47\% y en El Salvador a 36\%. Por el contrario, la importancia de las familias extensas o compuestas, aumenta del $20 \%$ y el $23 \%$ en Costa Rica y Chile, respectivamente; al 31\% en El Salvador. Tercero, en Chile y Costa Rica el Estado invierte una considerable cantidad de recursos por habitante en política social (US\$ 936 y 700 en promedio entre el 2000 y el 2005, en dólares de 1997), mientras que en El Salvador no (US\$ 82) ${ }^{3}$.

\section{CUADRO 3 \\ RED SOLIDARIA (EL SALVADOR)}

Este programa inició en el 2005 bajo la administración de Antonio Saca (2005-2009). Inicialmente, su población objetivo fueron los niños/as de comunidades rurales de hasta 13 años, pero poco después se amplió a todas las familias residentes con niños menores de 15 años que no hayan completado $6^{\circ}$ grado de primaria. La transferencia de dinero es condicionada a la asistencia escolar es de niños/as, con un tope de 2 a 3 por familia, y esta se entrega a las madres que deben de asegurar la asistencia escolar $y$ los controles de salud (uno, otro $u$ ambos dependiendo de la edad de niños/as); además de participar, ellas mismas, en los programas de capacitación. Según el Gobierno de El Salvador, en el período 2005-2008, 83654 familias recibieron los bonos de salud y educación, los cuales oscilaron entre US\$ 15 y 20 mensuales y representaron un $0,023 \%$ del PIB en el 2006 (CEPAL, 2007). Además de las transferencias, el programa contempla ampliación de la oferta de servicios públicos a comunidades sin escuelas o servicios de salud, $y$ la capacitación a personas adultas mediante círculos de alfabetización y propuestas de capacitación productiva — estas últimas, con menor nivel de implementación-. Para implementar los componentes de TMC $y$ de seguimiento y apoyo familiar, Red Solidaria opera con base en la contratación de ONGs que trabajan con redes de promotores locales.

Fuentes: Castañeda (2008); Góchez (2008); Gobierno de El Salvador, sitio web: <http://www.redsolidaria.gob.sv> [consultado el 07/07/2009].
Ver detalle de las fuentes y los resultados estadísticos en Martínez Franzoni (2008).
En Chile, las políticas sociales enfatizan la productividad laboral y un manejo mercantil de los riesgos sociales excepto entre las personas pobres, a quienes el estado provee de bienes y servicios básicos. En Costa Rica, en cambio, el estado provee servicios sociales a un amplio sector de la población, incluyendo a sectores medios, en gran medida, aunque no solo, mediante criterios relacionados con el trabajo formal asalariado. Por eso les llamamos regímenes productivista o mercantil (Argentina y Chile) y proteccionista o estatal (Brasil, Costa Rica, Panamá, México y Uruguay), respectivamente. El tercer régimen tiene políticas públicas con escasas capacidades y, más bien por omisión que por acción, las políticas públicas promueven una alta participación del trabajo femenino no remunerado $y$ de estrategias familiares para el manejo de los riesgos sociales. Este régimen familiarista se extiende a Bolivia, Colombia, Ecuador, El Salvador, Guatemala, Honduras, Nicaragua, Paraguay, Perú y República Dominicana (Martínez Franzoni, 2008).

¿En qué medida los programas de transferencias condicionadas afectan de forma distinta las relaciones de género? Si hubiera diferencias, ¿en qué medida esto ocurre debido a características de los respectivos regímenes de bienestar -en este caso no habría mucho que hacer más que cambiar el régimen-o a aspectos de diseño de los programas - en cuyo caso los márgenes de acción para promover transformaciones podrían ser mayores-?

\section{METODOLOGÍA}

A continuación explicamos en qué consiste el instrumento que hemos elaborado para analizar las relaciones entre los regímenes de bienestar y las relaciones de género, y que en este artículo aplicamos a la política pública, en particular a un programa social. Como se fundamentará, la principal fortaleza de este instrumento es que permite aprehender la desigualdad de género de manera multidimensional. Al hacerlo, permite no solo desglosar el análisis sino también las recomendaciones de políticas. 
3.1. INSTRUMENTO PARA EL ANÁLISIS EMPÍRICO: DIMENSIONES Y SUBDIMENSIONES

El instrumento teórico-metodológico que proponemos se enfoca en tres estructuras sociales en las que tienen lugar las relaciones de género (Walby, 1997), y que coinciden con las principales prácticas de asignación de recursos que dan lugar a los regímenes de bienestar: el trabajo remunerado, las políticas públicas, y el ámbito doméstico. Para determinar si los mayores o menores grados de igualdad de género existentes en cada una de estas estructuras, abordamos dimensiones propuestas por Nancy Fraser (1994): pobreza, explotación, marginación, desigualdad (de ingresos, servicios y uso del tiempo) y desvalorización social. Concretamente, nos interesa determinar en qué medida un programa social contribuye a reducir o a eliminar cada una de las cinco dimensiones (desde la pobreza, hasta la desvalorización social) ${ }^{4}$.

A partir de la combinación entre estructuras sociales $y$ dimensiones de análisis, el instrumento propuesto nos permite abordar la igualdad de género de manera multidimensional. No esperamos encontrar un régimen de bienestar que sea completamente igualitario o completamente desigual en cuanto a las relaciones de género en que se asienta $y / 0$ promueve. De acuerdo con Fraser, estamos frente a grados de igualdad/desigualdad, y no a su presencia o a ausencia a secas. Segundo, un régimen de bienestar que muestre un buen desempeño en alguno de los principios requeridos para la igualdad de género, no necesariamente lo tendrá con respecto a otros (Fraser, 1994). Por ejemplo, algunos regímenes de bienestar pueden tener un buen desempeño en cuanto al principio de anti pobreza, pero malo en cuanto al principio de equidad en los ingresos entre hombres y mujeres. Esto es consistente con la idea de que estamos ante constelaciones de prácticas, antes que índices aditivos.

$4 \quad$ Fraser propone un principio más, el anti androcentrismo, que nosotros consideramos como balance de las relaciones de género en su conjunto.
A continuación detallamos cómo cada una de las dimensiones constitutivas de los regímenes de bienestar se relaciona con cada uno de los principios propuestos para explorar sus relaciones con el orden de género.

Anti pobreza: Implica establecer las condiciones de mercantilización de la fuerza de trabajo entre las mujeres pobres. También requiere considerar su acceso a las transferencias y los servicios públicos como mecanismo para crear grados de autonomía entre su bienestar y su acceso al mercado laboral. Dado que los cuidados son principalmente una responsabilidad femenina y se proveen de manera no remunerada, se requiere conocer si la política pública asume los cuidados como una responsabilidad privada 0 , por el contrario, tiene un papel activo en delegar el cuidado, desde las familias a distintos ámbitos colectivos.

Anti explotación: Incluye las condiciones en que tienen lugar la participación laboral femenina, como es el caso de una vida laboral libre de acoso sexual. Conlleva determinar en qué medida la desmercantilización tiene lugar a partir de supuestos maternalistas mediante los cuales las mujeres acceden a los recursos públicos única o principalmente en su calidad de madres, las responsabilidades de cuidado se consideran exclusivamente suyas, $y$ los programas sociales reproducen y refuerzan estos supuestos. Implica considerar la violencia doméstica, tanto en términos de marcos normativos que considerarla como un asunto público, como en la aplicación efectiva de las leyes.

Anti marginalización: Requiere identificar grados de confinamiento a trabajos "femeninos". Conlleva abordar el grado de segregación ocupacional; las condiciones simbólicas y materiales a través de las cuales la política pública promueve medidas dirigidas a las madres solas o jefas de hogar, $y$ en qué medida hay presencia de infraestructura social de cuidados - sea que involucre al Estado, a las comunidades, o a otras instituciones como las agencias internacionales-; $y$ el grado en que efectivamente trasladan cuidados fuera del ámbito doméstico.

Anti desigualdad del ingreso monetario y social: remite a las brechas de remuneraciones por igual trabajo entre hombres $y$ mujeres; a las 
brechas en el ingreso social —es decir, en las transferencias y servicios públicos, principalmente estatales aunque no sólo- que a su vez permiten distintos grados y tipos de desmercantilización; $y$ las brechas de ingreso producidas a raíz de la disolución formal o práctica de las parejas. Del uso del tiempo: consideramos la demanda de trabajo femenino no remunerado demandado por los programas sociales, en el entendido de que a medida que esta demanda aumenta, aumenta también la carga total de trabajo femenino.

De la valoración social: más allá de los ingresos, ¿son las mujeres y sus habilidades laborales, igualmente valoradas que las habilidades asociadas al trabajo masculino?; $y$ alude al reconocimiento del trabajo doméstico como un criterio para acceder a la política pública, diferente a la dependencia económica de la pareja.

\section{CUADRO 4}

REGÍMENES DE BIENESTAR Y ÓRDENES DE GÉNERO: DIMENSIONES Y SUBDIMENSIONES A CONSIDERAR EN EL ANÁLISIS DE LOS PROGRAMAS DE TRANSFERENCIAS CONDICIONADAS

\begin{tabular}{|c|c|c|c|}
\hline PRINCIPIOS & TRABAJO REMUNERADO & POLÍTICA PÚBLICA & $\begin{array}{l}\text { PRODUCCIÓN } \\
\text { DOMÉSTICA }\end{array}$ \\
\hline POBREZA & $\begin{array}{l}\text { Acceso al trabajo } \\
\text { remunerado }\end{array}$ & $\begin{array}{l}\text { Afirmación positiva en el } \\
\text { acceso a la política social }\end{array}$ & \\
\hline EXPLOTACIÓN & $\begin{array}{l}\text { Jornadas y estabilidad } \\
\text { Prevención y sanción del } \\
\text { acoso sexual }\end{array}$ & $\begin{array}{l}\text { Superación de supuestos } \\
\text { maternalistas }\end{array}$ & $\begin{array}{l}\text { Carga total de trabajo } \\
\text { femenino } \\
\text { Vida libre de violencia }\end{array}$ \\
\hline MARGINACIÓN & Desegregación laboral & $\begin{array}{l}\text { Acciones afirmativas para } \\
\text { acceder a servicios sociales }\end{array}$ & $\begin{array}{l}\text { Decisiones sobre el uso de } \\
\text { los recursos }\end{array}$ \\
\hline $\begin{array}{l}\text { DESIGUALDAD EN EL } \\
\text { USO DEL TIEMPO }\end{array}$ & & $\begin{array}{l}\text { Demanda de } \\
\text { reorganización de roles en } \\
\text { el ámbito doméstico }\end{array}$ & $\begin{array}{l}\text { Reorganización de uso del } \\
\text { tiempo }\end{array}$ \\
\hline $\begin{array}{l}\text { DESIGUALDAD EN EL } \\
\text { INGRESO MONETARIO Y } \\
\text { SOCIAL }\end{array}$ & \multicolumn{2}{|c|}{ Reducción de brechas de ingreso } & \\
\hline DESVALORACIÓN SOCIAL & $\begin{array}{l}\text { Valoración del trabajo y las } \\
\text { capacidades femeninas }\end{array}$ & $\begin{array}{l}\text { Trato y paradigma } \\
\text { igualitario por parte de } \\
\text { funcionarios/as públicos }\end{array}$ & \\
\hline
\end{tabular}

\subsection{PRINCIPALES PROPOSICIONES}

A continuación recurrimos a la herramienta metodológica propuesta para explorar cómo los programas de transferencias monetarias condicionadas, inciden en la desigualdad de género. ¿Es posible decir que la reproducen, acentúan y/o reducen? Con este objetivo, a partir de la literatura existente sobre los TMC, planteamos un conjunto de proposiciones relacionadas con las dimensiones y los principios resumidos previamente (cuadro 4). Así, el cuadro 5 presenta las principales proposiciones que analizaremos empíricamente (un total de 17), según los efectos positivos o negativos esperados con respecto a los objetivos explícitos (la superación de la pobreza), o implícitos (concretamente relacionados con la equidad de género) estos programas. El formato en que formulamos estas proposiciones combina el efecto esperado, con su justificación.

La formulación de proposiciones nos permite transitar desde el nivel teórico al análisis empírico. Nos facilita además confrontar los distintos aspectos del problema con la respectiva evidencia empírica. Finalmente, y desde el 
CUADRO 5

TMC: PRINCIPALES PROPOSICIONES A ANALIZAR SEGÚN OBJETIVOS

\begin{tabular}{|c|c|c|c|c|}
\hline & \multicolumn{2}{|c|}{ PRINCIPALES: SUPERACIÓN DE LA POBREZA } & \multicolumn{2}{|c|}{ SECUNDARIOS: EQUIDAD DE GÉNERO } \\
\hline & EFECTOS POSITIVOS & EFECTOS NEGATIVOS & EFECTOS POSITIVOS & EFECTOS NEGATIVOS \\
\hline 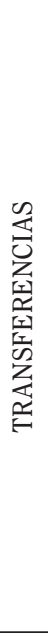 & $\begin{array}{l}\text {-Asignan recursos } \\
\text { con mayores grados } \\
\text { de autonomía que los } \\
\text { programas históricos } \\
\text { de transferencias y } \\
\text { hay criterios explícitos } \\
\text { conocidos por la } \\
\text { población. } \\
\text {-Aumentan las } \\
\text { expectativas que tiene la } \\
\text { población con respecto al } \\
\text { papel del Estado. }\end{array}$ & $\begin{array}{l}\text {-Dado enfoque asistencial, } \\
\text { conlleva gratitud antes } \\
\text { que fortalecimiento de } \\
\text { ciudadanía. } \\
\text {-Hay una tensión } \\
\text { entre condicionalidad } \\
\text { y la mayor autonomía } \\
\text { económica de las } \\
\text { personas adultas. } \\
\text {-Por un lado se invierte } \\
\text { en las niñas pero por el } \\
\text { otro se reproduce papel } \\
\text { tradicional de las madres. }\end{array}$ & $\begin{array}{l}\text {-Las transferencias ( } y \\
\text { otros componentes como } \\
\text { la capacitación), permiten } \\
\text { mayor autonomía, estatus } \\
\text { y autoestima. } \\
\text {-Visibilizan el papel } \\
\text { central que tienen las } \\
\text { mujeres en el cuidado y } \\
\text { la administración de los } \\
\text { recursos de la familia. }\end{array}$ & $\begin{array}{l}\text {-No promueve } \\
\text { empoderamiento, lo cual } \\
\text { requeriría la generación } \\
\text { de ingresos propios. } \\
\text {-Responsabilizan a las } \\
\text { mujeres de gestionar la } \\
\text { pobreza sin alterar la } \\
\text { división sexual del trabajo } \\
\text { en el hogar. } \\
\text {-Desatienden } \\
\text { posibles efectos de las } \\
\text { transferencias en la } \\
\text { violencia doméstica. } \\
\text {-Aumentan las tensiones } \\
\text { por el uso del tiempo. } \\
\text {-Cuando promueve } \\
\text { participación, es solo } \\
\text { individual. }\end{array}$ \\
\hline  & $\begin{array}{l}\text {-Mejoran el acceso a } \\
\text { los servicios (matrícula } \\
\text { y asistencia escolar } \\
\text { y controles de } \\
\text { salud, incluyendo a } \\
\text { embarazadas). }\end{array}$ & $\begin{array}{l}\text {-Las niñas tendrán las } \\
\text { mismas limitaciones } \\
\text { estructurales que hoy } \\
\text { tienen sus madres } \\
\text { para acceder a trabajo } \\
\text { remunerado de calidad. } \\
\text {-Los programas llegan a } \\
\text { lo sumo a la capacitación, } \\
\text { pero no dan el salto a la } \\
\text { articulación productiva y } \\
\text { en materia de empleo. }\end{array}$ & $\begin{array}{l}\text {-Incentivan que las } \\
\text { niñas tengan acceso a } \\
\text { la educación y reducen } \\
\text { brechas entre varones y } \\
\text { mujeres en la asistencia } \\
\text { escolar. }\end{array}$ & $\begin{array}{l}\text {-Promueven equidad } \\
\text { entre las niñas pero } \\
\text { maternalismo entre las } \\
\text { adultas que solo son } \\
\text { "correas de trasmisión", } \\
\text { no beneficiarias. }\end{array}$ \\
\hline
\end{tabular}

Fuente: Elaboración propia, con base en Molyneux (2006; 2007)

punto de vista de las implicaciones que el análisis tiene para la reformulación de programas, emplear proposiciones nos permite pensar en escenarios según las dimensiones de la desigualdad que se quiera priorizar.

Dado que los programas analizados se encuentran fuertemente insertos en ambientes internacionales de política, esperamos encontrar múltiples aspectos comunes atribuibles a procesos similares de formación de política pública. Sin embargo, esperamos también encontrar importantes diferencias, asociadas a la articulación de los programas de transferencias condicionadas con el resto del régimen de política social, por un lado, y con los mercados laborales y la organización de las familias, por el otro, en tanto constelaciones de relaciones particulares a cada régimen de bienestar.

\subsection{LOS DATOS DISPONIBLES}

Como material empírico nos basamos en fuentes secundarias (análisis de los programas) y primarias (entrevistas y grupos focales) ${ }^{5}$. Empleamos dos tipos de fuentes: secundarias, a

5 Ver Martínez Franzoni, Juliana y Koen Voorend (2008). 
partir de todos los estudios que pudimos identificar para cada uno de los tres programas, $y$ primarias, a partir de entrevistas (Chile y El Salvador) y grupos focales (El Salvador). En el caso de Chile Solidario disponemos de una cantidad $y$ variedad importante de estudios realizados desde fuera del Estado. Dada la riqueza de las fuentes secundarias disponibles, realizamos entrevistas pero no grupos focales. En el caso de Red Solidaria nos basamos en cuatro estudios realizados por consultores externos contratados por el programa, $y$ de grupos focales realizados en el marco de nuestro estudio. En el caso de Avancemos solo contamos con un estudio realizado por un órgano de control del Estado costarricense. En este último caso, por circunstancias de la coyuntura que vincularon al programa y a sus jerarcas con el presunto uso incorrecto de fondos, conllevó la renuncia de aquellos y en general, mucha reticencia a hablar públicamente del programa. Esperamos que en los próximos seis meses, a medida que el escenario político vuelva a la normalidad, podamos completar el estudio de este programa, tanto a partir de entrevistas como de grupos focales. A los efectos del presente análisis, complementamos el estudio de caso con análisis de los dos periódicos de mayor circulación del país.

Para cada dimensión presentamos principales proposiciones que plantea la literatura (frecuentemente contradictorias) y el balance que hacemos a la luz de la evidencia empírica disponible y que detallamos en otro documento (Martínez y Voorend, 2008).

\section{TMC E IGUALDAD DE GÉNERO: ¿MÁS, MENOS O DEPENDE?}

A partir del instrumento propuesto $y$ de las 17 proposiciones a que dio lugar, valoramos en qué medida las TMC - Chile Solidario en Chile, Avancemos en Costa Rica y Red Solidaria en El Salvador - promueven o inhiben la equidad de género en cada uno de los tres países considerados.

A los efectos de simplificar la presentación de hallazgos, agrupamos proposiciones según se relacionen con cada uno de los principios considerados. Para uno resumimos resultados positivos y negativos esperados, $y$ presentamos un balance de la evidencia recogida.

\subsection{ACCESO AL TRABAJO REMUNERADO (PRINCIPIO DE ANTI POBREZA)}

Proposiciones a partir de la literatura: Las TMC permiten algún grado de acceso desmercantilizado (estatal), a los ingresos monetarios. Sin embargo, el enfoque de formación de capital humano enfatiza la oferta $y$ no la demanda de fuerza de trabajo, y las niñas y los niños que hoy reciben transferencias, en el futuro enfrentarán las mismas limitaciones estructurales para acceder a los mercados laborales que enfrentan sus madres $y$ padres. Los programas llegan a lo sumo a la capacitación, pero no dan el salto a la articulación productiva $y$ en materia de empleo.

Balance empírico: En ninguno de los tres casos, los programas inciden claramente en las condiciones para el acceso al mercado laboral. Las acciones generalmente giran en torno al ámbito de la política social y no de la política laboral. Los programas dan por sentado que el empleo, de alguna manera, será generado pero no por acciones llevadas a cabo desde los programas. Sin embargo, hay variaciones en cómo abordan su articulación con el empleo. El Chile Solidario es el que más decidida y explícitamente busca promover la inserción laboral. Además, aunque de manera insuficiente, contempla la existencia de servicios de cuidados que facilite dicha inserción entre las mujeres. Aunque no promueve una reorganización de los roles de género, sí reconoce, al menos formalmente, que la inserción laboral femenina conlleva atender demandas de cuidados, en particular de la población infantil. Red Solidaria contempla, aunque sea formalmente, la capacitación y la inserción productiva. Ha llevado a cabo algunas acciones en materia de capacitación, aunque no ha logrado su articulación con iniciativas productivas. Además, los cuidados y el trabajo doméstico no remunerado, en ningún momento se separan y dejan de ser responsabilidad materna. Avancemos se enfoca casi exclusivamente en la dimensión educativa. Sin embargo, 
en el mediano plazo, el estímulo económico a jóvenes destacados/as para que estudien carreras para las cuales haya demanda insatisfecha de empleo, podría incidir en una mejor inserción laboral de los/as jóvenes. Aunque las variaciones entre programas reflejan diferencias de diseño que podrían ser corregidas, el dar el paso a la creación de empleo conlleva alteraciones más estructurales en los respectivos regímenes de bienestar que podrían ser más fáciles de promover en Costa Rica (dado el tipo de intervención que actualmente tiene el Estado en materia social) que en El Salvador o Chile.

\subsection{AFIRMACIÓN POSITIVA EN EL ACCESO A LA POLÍTICA SOCIAL (PRINCIPIOS DE ANTI POBREZA Y ANTI MARGINACIÓN)}

Proposiciones a partir de la literatura: Permiten mayores grados de autonomía, estatus y autoestima entre las mujeres. Hay acuerdo en que sean las mujeres quienes reciban las transferencias. Además, las contraprestaciones pueden ampliar el acceso de las mujeres a servicios sociales. Mejoran el acceso y/o la retención de niños/as y jóvenes en el sistema educativo y los controles de salud; también de las mujeres embarazadas y en algunos casos, mediante controles ginecológicos periódicos. Sin embargo, a la vez que promueven la equidad para las niñas, solo se dirigen a las mujeres en tanto madres para llegar a niños/as y en tanto beneficiarias de medidas asociadas a los programas.

Balance empírico: Tienen en común que al entregar las transferencias a las mujeres, reconocen su importancia en la administración de los recursos del hogar. Los hacen con distintos grados: muy enfatizado y publicitado en El Salvador y Chile, menos en Costa Rica. También tienen en común el exigir una contraprestación que, cómo es definida, favorece la ampliación del acceso a servicios sociales por parte de las mujeres adultas, tanto en Chile como El Salvador, no así en Costa Rica, $y$ mediante una oferta de transferencias y servicios mucho más amplia en Chile que en El Salvador. La entrega del dinero tiene lugar de la mano de nociones relativas el qué debe ser gastado generalmente en alimentos en El
Salvador y útiles o materiales relacionados con la asistencia escolar en Chile y Costa Rica.

La afirmación positiva que se realiza en el acceso a los servicios de educación y de salud es en términos socioeconómicos y no de género: las transferencias se dirigen indistintamente a niños y a niñas. Sin embargo, generalmente las mujeres alcanzan mayor acceso que los hombres (ver siguiente proposición). El rango de servicios es mínimo en El Salvador y máximo en Chile. Dada la escasa cobertura previa de servicios sociales en las comunidades en que se ejecuta Red Solidaria, es este país, en donde se evidencia que el programa ha conllevado a una ampliación de acceso a los servicios sociales, aún cuando sea de servicios básicos y frecuentemente inestables (como ocurre con algunos controles de salud que son periódicos pero algo erráticos). Dado que estas no distinguen entre niños y niñas, es de esperar que estén contribuyendo al acceso de unos $y$ otras.

\subsection{REDUCCIÓN DE BRECHAS SOCIOECONÓMICAS EN EL ACCESO AL INGRESO SOCIAL (PRINCIPIO DE IGUALDAD EN EL INGRESO)}

Proposiciones a partir de la literatura: Estos programas aumentan las expectativas que tiene la población con respecto al papel del Estado en la reasignación de recursos. Sin embargo, el enfoque asistencial en el que se asientan, tiende a generar entre quienes las reciben, muestras de gratitud antes que un fortalecimiento de la ciudadanía o de la noción de derechos.

Balance empírico: Los tres programas logran efectivamente reducir brechas socioeconómicas en el acceso al ingreso social. Sin embargo, esta reducción es cualitativamente distinta entre programas debido a los regímenes de bienestar en los que se insertan. La población que accede al Chile Solidario tenía ya acceso a servicios sociales, $y$ algo similar estaría ocurriendo en Costa Rica. En cambio, mediante Red Solidaria el Estado ha ampliado su alcance a la población rural previamente carente de dichos servicios, aún cuando persisten limitaciones en infraestructura, recursos humanos, calidad y canasta de servicios. Dado que históricamente la población tuvo escasa o nula 
experiencia con un Estado redistribuidor, el aprovechamiento político del programa, asociado más a la noción de "ayuda" que de derechos, incide fuertemente en cómo la población está recibiendo las transferencias y los servicios. Así, pareciera que en Chile el acceso se inserta en expectativas preexistentes con respecto al papel del Estado, mientras que en El Salvador tiende a fortalecer una percepción de agradecimiento a una persona, el Presidente de la República, en tanto figura política asociada al programa. En Costa Rica, mientras tanto, Avancemos es un programa muy puntual en el marco de un Estado redistribuidor $y$ de una población con altas expectativas con respecto a su papel. Sea que se inserte en un entorno que vincula el programa con derechos o con la asistencia $y$ la caridad del Estado, Avancemos, al igual que Red Solidaria aunque posiblemente en menor medida que Chile Solidario, al margen de los criterios empleados para seleccionar beneficiarios/as y de su carácter más o menos técnico, el rédito político es para las respectivas administraciones de gobierno.

\subsection{SUPERACIÓN DE SUPUESTOS MATERNALISTAS (PRINCIPIO DE ANTI EXPLOTACIÓN)}

Proposiciones a partir de la literatura: Mediante su componente de transferencias estos programas visibilizan el papel central de las mujeres en los cuidados de niños y niñas. Aunque se invierte en las niñas, el Estado refuerza el papel tradicional de las madres en el ámbito doméstico. Primero, la demanda de la contraprestación es generalmente a las mujeres, $y$ son ellas quienes deben destinarle tiempo. Segundo, las medidas efectivamente dirigidas a las mujeres adultas, se relacionan con su condición de madres y cuidadoras, antes que de trabajadoras (mucho menos ciudadanas).

Balance empírico: $\mathrm{El}$ reconocimiento al papel de las mujeres en el ámbito doméstico que trae aparejada la entrega de las transferencias, viene acompañado de maternalismo en las contraprestaciones y los servicios disponibles para las mujeres. Si bien los programas, en particular Red Solidaria, contemplan la participación de los hombres como titulares o corresponsables, en la práctica su presencia es casi inexistente. Además, desde los servicios se atienden a las mujeres en función de su papel reproductivo (principalmente control de embarazo, parto y post parto en el caso de la salud) o de cuidados (caso de otros servicios incluidos en el Chile Solidario). Así, el grado de maternalismo es mayor en El Salvador y menor en Chile. Dado que la ausencia de estos servicios conlleva a importantes costos para las propias mujeres, representa un claro avance, el que los programas de TMC amplían estos servicios y midan resultados como ocurre en El Salvador. No obstante, no debería excluir medidas que mejoren el acceso a ingresos propios, para lo cual sería necesario un mayor abanico de servicios orientados, para mencionar algunos, a la educación, los cuidados infantiles y el crédito.

\subsection{DEMANDA DE REORGANIZACIÓN DE ROLES (PRINCIPIO DE IGUALDAD EN EL USO DEL TIEMPO)}

Proposiciones a partir de la literatura: $\mathrm{La}$ condicionalidad —es decir, la contraprestación que se exige a cambio de la transferencia monetaria- podría en teoría usarse para promover una transformación de la división sexual del trabajo. Sin embargo, los programas refuerzan la exclusiva responsabilidad de las mujeres en la gestión de los escasos recursos disponibles, $y$ deja intacta la organización tradicional del trabajo doméstico y los cuidados. Al hacerlo, los programas aumentan las ya importantes tensiones que experimentan las mujeres en el uso de su tiempo.

Balance empírico: El paradigma es común a los tres programas y está orientado al aprovechamiento antes que a la transformación de la división sexual del trabajo. Esta demanda puede o no generar tensiones en el uso del tiempo femenino, según los entornos laborales en los que se insertan los programas. La tensión es mínima en El Salvador porque las madres rurales a los que se dirige generalmente carecen de trabajo remunerado. Más bien, en muchos casos, conciben el programa como una oportunidad para desarrollar capacidades que luego les permitan tener ingresos propios. 
Si bien consideran que el programa les lleva a interrumpir las tares domésticas, generalmente consideran que vale la pena hacerlo. En Avancemos, la contraprestación no exige tiempo sino solo controles de asistencia escolar de las personas jóvenes.

\subsection{TRATO Y PARADIGMA IGUALITARIO POR PARTE DE FUNCIONARIOS/AS PÚBLICOS (PRINCIPIO DE IGUALDAD EN LA VALORACIÓN SOCIAL)}

Proposiciones a partir de la literatura: Permiten una asignación de los recursos al margen del clientelismo propio de los programas históricos de transferencias: hay criterios explícitos y la población los conoce. Sin embargo, perduran criterios asistencialistas y de "ayuda social" - por oposición a criterios relacionados con los derechos - en el acceso a los programas sociales por parte de estas personas.

Balance empírico: Dado que los programas están diseñados con base en el aprovechamiento de la división tradicional y jerárquica del trabajo entre hombres y mujeres, existe una suerte de conflicto con usar el condicionamiento para reorganizar roles en el ámbito doméstico. El hacerlo implicaría darle menos importancia a lo que ahora es el objetivo central de estos programas (vale decir, la formación de capital humano de niños y niñas), y más importancia a procesos de transformación de tareas en el ámbito doméstico por parte de la población adulta. Desde este punto de vista, a lo más que pueden llegar estos programas es a desplegar un "feminismo de la diferencia", que valora socialmente a las mujeres pero desde aquellas tareas asociadas a la reproducción y a los cuidados. Sin embargo, en general, aunque más evidente en Red Solidaria, existe una desvalorización social dado que las madres "no saben" con respecto a un rango relativamente amplio de actividades (desde higiene de alimentos hasta derechos sexuales y reproductivos), como sí saben los/as funcionarios/as que las atienden. A la vez, la manera en que un paradigma tradicional del reparto de tareas entre hombres $y$ mujeres se lleva a la práctica, así como la homogeneidad o "fisuras" en su interpretación, varía según las capacidades técnicas e institucionales que el Estado tenga y en función de esta, según su intervención sea directa (como en Costa Rica) o indirecta, es decir mediante organizaciones no gubernamentales (como en El Salvador).

\section{CONCLUSIONES}

En general, en términos de su diseño, hay un alto grado de homogeneidad entre programas. Primero, los tres promueven una afirmación positiva en el acceso de las mujeres a la política social, en particular mediante el acceso a las transferencias. Por su parte, el acceso a los servicios de educación y de salud, se promueve de manera universal, tanto entre niños como entre niñas. Segundo, se asientan en supuestos maternalistas y solo de manera relativamente marginal contemplan o promueven a las mujeres como trabajadoras $y$, todavía en menor medida, a los hombres como cuidadores. Tercero, y por lo tanto, ninguno de los programas promueve una reorganización de roles en el ámbito doméstico. En todo caso, el programa deja librado dicha reorganización a preferencias de las familias. En ausencia de incentivos, dichos cambios son poco probables. Más bien los programas parten $y$ aprovechan capacidades genéricamente construidas, en particular el que las mujeres sepan gestionar mejor los recursos y el cuidado de niños/as. Por eso, y en quinto lugar, no sin cierto sesgo tecnocrático en términos de quiénes "saben" hacer qué, estos programas valoran a las mujeres en tanto madres pero no necesariamente en tanto ciudadanas o trabajadoras. Finalmente, en la práctica tienen una débil articulación con el empleo $y$ el fomento productivo $y$ cuando lo intentan, el acceso al empleo se concibe como producto de mejoras en el capital humano de la oferta antes que de la demanda de trabajo.

Las diferencias importantes entre programas aluden, primero, al grado en que han explicitado su articulación con el empleo y la producción. Esta es mayor en Chile Solidario y en Red Solidaria que en Avancemos. Segundo, existen diferencias en el grado en que los programas se dirigen a las mujeres adultas en sus propios términos, más allá de recibir las transferencias o garantizar las medidas a las que 
estas se condicionan. Sin embargo, este involucramiento de las mujeres adultas es también mayor en Chile Solidario y Red Solidaria que en Avancemos. Tercero, en general las mujeres se suman al programa en tanto madres, pero hay diferencias en el grado en que el programa contempla la presencia de mujeres trabajadoras (Chile Solidario) o de hombres cuidadores (Red Solidaria). Cuarto, existe una diferencia en la amplitud de transferencias y servicios a los que tienen lugar quienes acceden al programa y por lo tanto, en los grados de desmercantilización de las necesidades sociales que permiten cada programa, mayor en Chile $y$ menor en El Salvador. De todas las diferencias identificadas, esta tiene directa relación con el régimen de bienestar en el que se insertan los programas. Mientras que el Chile Solidario se inserta en el marco de un régimen con Estado fuerte e inversión social comparativamente alta, Red Solidaria se inserta en un régimen familiarista y con inversión social muy baja. Quinto, se presentan diferencias en la tensión por el uso del tiempo de las mujeres, que es mayor en zonas urbanas, porque es más frecuente que estas tengan trabajo remunerado, mientras que en zonas rurales, esa frecuencia es menor. Sexto, varía el grado en que la implementación del programa la hace propiamente funcionarios/as públicos, en comparación a gobiernos locales y a organizaciones no gubernamentales. Esto último es mayor en El Salvador, menor en Costa Rica, e intermedio en Chile. Independientemente de lo que se diga en el papel, cuanto mayor sea la diversidad de actores que participan, mayor será también la heterogeneidad de visiones desplegadas en materia de relaciones de género.

La relativa homogeneidad entre programas insertos en regímenes de bienestar altamente contrastantes, indicaría que esta se debe más a aspectos de diseño de los programas, que de articulación de las políticas sociales con los mercados laborales y las familias. En segundo lugar, indicaría que es posible introducir cambios en dichos diseños, que permitan promover mayores grados de igualdad de género en todas las dimensiones consideradas. Tercero, esto sería posible sin dejar a un lado los objetivos actuales explícitos relativos al mejoramiento de la formación de capital humano en niños/as y jóvenes, aunque requeriría jerarquizar aquellos relacionados con la igualdad. Si hubiera que priorizar, dos serían los "nudos" a desatar para promover mayores grados de igualdad de género. El primero tiene que ver con la mayor autonomía económica de las mujeres, mediante una mejor articulación de estos programas sociales, con el mercado laboral y la producción. El segundo aspecto alude a un papel más activo del Estado en promover la reorganización de roles en el ámbito doméstico, tanto por una mayor disponibilidad de servicios de cuidados, como, deseablemente, aunque más difícil, por una mayor presencia de hombres cuidadores.

En el balance de las distintas dimensiones de análisis, consideramos que los programas de transferencias condicionadas afectan las relaciones de género de una manera que no admite blancos ni negros, sino grises. Y es precisamente a partir de estos grises que las TMC podrían reformularse para promover en lugar de reforzar la desigualdad de género. El hacerlo implicaría cambios del programa "hacia adentro", es decir, del propio diseño, $y$ del programa hacia afuera, por la articulación con otras políticas (sociales pero también laborales y productivas). La principal decisión política que se requiere es la de darle a las mujeres la misma importancia que los programas dan hoy a niños, niñas y jóvenes. Para algunos/as, esta preocupación por las mujeres y por la igualdad de género es y será una cuestión de principios. Para otros/as, en cambio, será instrumental al cumplimiento de los objetivos que en el mediano plazo se busca alcanzar para quienes, siendo niños/as jóvenes hoy, serán personas adultas mañana. En uno u otro caso, sería deseable y posible que desde los programas de transferencias condicionadas se definirían "rutas críticas" para correr las fronteras de la pobreza, la explotación, la marginación, la desigualdad y la desvalorización. Dichas rutas necesariamente reflejarán el escenario de política social propio de cada país según el régimen de bienestar en el que se inserta cada programa. 
BIBLIOGRAFÍA

Barba, Carlos ¿Combatir la pobreza o construir ciudadanía para todos? América Latina. Regimenes de bienestar en transición al iniciar el siglo XXI. Guadalajara: Universidad de Guadalajara, 2007.

Brownmiller, Susan . Against Our Will: Men, Women, and Rape. New York: Simon \& Schuster, 1975.

Castañeda, Tarsicio. Red Solidaria de El Salvador: avances y retos para el futuro. Programa Red Solidaria, 2008a. [Informe en línea] En: <http://www.redsolidaria. gob.sv/component/option,com_docman/ task,cat_view/gid,42/> [consultado agosto, 2008].

Castañeda, Tarsicio. Red Solidaria de El Salvador: Avances y retos para el futuro. Programa Red Solidaria. 2008b. [PPT en línea] En: <http://www.redsolidaria.gob. sv/component/option,com_docman/ task,cat_view/gid,42/> [consultado agosto, 2008].

CEPAl. Panorama social de América Latina 2007. Santiago de Chile: CEPAL. Noviembre, 2007.

CEPAL. Panorama social de América Latina 2006. Santiago de Chile: CEPAL. Diciembre, 2006.

Cohen, Ernesto y Rolando Franco (editores). Transferencias con corresponsabilidad: una mirada latinoamericana. México: FLACSO, 2006.

Cohen, Ernesto y Villatoro Pablo. "Chile: Puente-Chile Solidario”. Cohen, Ernesto $y$ Rolando Franco. Transferencias con corresponsabilidad. Una mirada latinoamericana. FLACSO-México, 2006: 179-226.
Contraloría General de la República. "Informe nro. DFOE-SOC-17-2008: Informe sobre el diseño y ejecución del programa de transferencia monetaria condicionada denominado Avancemos". Documento mimeografiado. Setiembre, 2008.

Crompton, Rosemary. "Reviewed Work(s): Gender Transformations by Sylvia Walby". The British Journal of Sociology 49 (4). Blackwell Publishing, 1998: 678679 .

Diario Extra. "Zumbado inició gira por colegios públicos". 19-02-2008. En: <http:// www.diarioextra.com/2008/febrero/19/ nacionales02.php $>$. [consultado octubre, 2008].

Diario Extra. "Compran celular con dinero que les dan para el colegio". 20-07-2007. En: <http://www.diarioextra.com/2007/ julio/20/opinion01.php >. [consultado octubre, 2008].

Duke, Karen. Reporte de grupos focales, Proyecto Regímenes de Bienestar y Variedades de Patriarcado en América Latina. 30 de julio. El Salvador. 2008: 14.

Esping-Andersen, Gøsta. The Three Worlds of Welfare Capitalism. Princeton: Princeton University Press, 1990.

Feitosa de Britto, Tatiana. "Los desafíos del programa de transferencias monetarias condicionadas en El Salvador, Red Solidaria”. Internacional Poverty Centre 9. Programa Red Solidaria. 2008. [Revista en linea] En: <http://www.redsolidaria. gob.sv/component/option,com_docman/ task,cat_view/gid,42/> [consultado agosto, 2008].

Ferre, María Ester. "El sistema de Chile Solidario: ¿Una política innovadora para erradicar la pobreza?" Riquelme, Verónica y María Elena Valenzuela (editoras). Chile 
Solidario y los desafíos de la igualdad. Proyecto género, pobreza y empleo. Oficina Internacional del Trabajo y Programa de las Naciones Unidas para el Desarrollo. 2005: 25-76.

Filgueira, Fernando. "El nuevo modelo de prestaciones sociales en América Latina: residualismo y ciudadanía estratificada". Roberts, Brian (editor). Ciudadanía y politica social. San José, Costa Rica. FLACSO/SSRC, 1998: 71-116.

Fraser, Nancy. "After the Family Wage: Gender Equity and the Welfare State". Political Theory 22 (4). Sage Publication, 1994: 591-618.

Galasso, Emanuela. "With their effort and one opportunity: Alleviating extreme poverty in Chile”. Banco Mundial. Conferences: Policy Seminar with Emanuela Galasso: Evaluation Results of Chile Solidario: With Their Effort and One Opportunity. 2006. [Artículo en línea] En: <http:// www.iadb.org/res/pub_desc.cfm?pub_ $\mathrm{id}=\mathrm{S}-1>$ [consultado setiembre, 2008].

Gallardo, Cecilia. Entrevista en el Diario de Hoy, 20/02/08. El Salvador. 2008. [Artículo en línea] En: <http://www.elsalvador.com/ noticias/2006/10/18/nacional/nac2.asp> [consultado marzo, 2008].

Gallardo, Cecilia. "Red Solidaria no es una bandera política, es un programa de país". Centroamérica 21. 2007. [Artículo en línea] En: <http://www.centroamerica21. com/web 21 / ed it / $25-2 / \mathrm{n}$ acion 2 . htmlhttp://www.centroamerica21.com/ web21/edit/25-2/nacion2.htmlhttp:// www.centroamerica21.com/web21/ edit/25-2/nacion2.html> [consultado abril, 2008].

Gallardo, Cecilia. "Red solidaria "atrapa" la pobreza”. El Diario de Hoy, 20/02/2008. [Artículo en línea] 2006. En: <http:// www.elsalvador.com/noticias/2006/10/18/ nacional/nac2.asp $>$ [consultado marzo, 2008].

Gallardo, Cecilia. Palabras contra la pobreza. FaroNet, 6/03/2005. [Artículo en línea] En: <http://www.elfaro.net/secciones/ noticias/20050307/noticias12_20050307. asp > [consultado marzo, 2008].

Gobierno de Chile. Chile Solidario. Sistema de Protección Social-Estadísticas. Programa Chile Solidario. 2009. [Artículo en línea] En: <http://www.chilesolidario.gov.cl/ est/est1.php> [consultado julio, 2009].

Gobierno de Chile. Analizan aportes de Chile Solidario al enfoque de género. Programa Chile Solidario. 2006. [Artículo en línea] En: <http://www.chilesolidario. gov.cl/publico/noticias.php?not=395> [consultado setiembre, 2008].

Gobierno de El Salvador. Red Solidaria llega a 100 municipios y 120 mil familias reciben bono para salud y educación. Programa Red Solidaria [Artículo en línea] En: < http://www.redsolidaria.gob. sv/content/view/857/> [consultado julio, 2009].

Góchez, Rafael. Percepción de los beneficiarios del funcionamiento e impacto de Red Solidaria. 2008. [Artículo en línea] En: <http://www.redsolidaria.gob.sv/ content/view/810/> [consultado agosto, 2008].

Gough, Ian y Wood, Geof (editores). Insecurity and welfare regimes in Asia, Africa and Latin America. Cambridge: Cambridge University Press, 2004.

Henríquez, Helia y Reca, Inés. "Propuestas para fortalecer los componentes de género en el sistema de protección Chile Solidario". Riquelme, Verónica y María Elena Valenzuela (editoras). Chile Solidario 
y los desafíos de la igualdad. Proyecto género, pobreza y empleo. Oficina Internacional del Trabajo y Programa de las Naciones Unidas para el Desarrollo. 2005: 77-156.

La Nación. "Zumbado se forja 'imagen de ministro de los pobres'”. 16-04-2008. [Artículo en línea] En: <http://www. nacion.com/ln_ee/2008/abril/16/ pais1495521.html> [consultado octubre, 2008].

La Nación. "29 000 alumnos dejaron aulas en el primer semestre del 2007". 28-11-2007. [Artículo en línea] En: <http://www. nacion.com/ln_ee/2007/noviembre/28/ pais1332603.html> [consultado octubre, 2008].

La Nación. "Oficina de diputada tramita bonos y becas". 19-02-2007. [Artículo en línea] En: < http://www.nacion.com/ ln_ee/2007/febrero/13/pais994599. html> [consultado octubre, 2008].

La Prensa Libre. "Universidades privadas darán 1000 becas a jóvenes del programa Avancemos". 30-01-2008. [Artículo en línea] En: <http://www.prensalibre. co.cr/2008/enero/30/nacionales04.php> [consultado octubre, 2008].

La Prensa Libre. "Pobreza extrema desafía el programa "Avancemos"”. 13-11-2007. [Artículo en línea] En: <http://www. prensalibre.co.cr/2007/noviembre/13/ nacionales09.php $>$ [consultado octubre, 2008].

Lewis, Jane. "Gender and the Development of Welfare Regimes". Journal of European Social Policy 2 (3). Sage Publications, 1992: 159-73.

MacKinnon, Catherine. Towards a Feminist Theory of the State. Cambridge: Harvard University Press, 1989.
Martínez Franzoni, Juliana. Domesticar la incertidumbre en América Latina: mercados laborales, politica social y familias. San José: Editorial de la UCR, 2008.

Martínez Franzoni, Juliana y Koen Voorend. "Transferencias condicionadas: ¿blancos, negros o grises?". Documento de trabajo. Observatorio de la Desigualdad, Centro de Estudios Latinoamericanos, Universidad de Miami. 2008.

Mauro, Amelia. "Familias y población de Chile Solidario". Riquelme, Verónica y María Elena Valenzuela (editoras). Chile Solidario y los desafíos de la igualdad. Proyecto género, pobreza y empleo. Oficina Internacional del Trabajo y Programa de las Naciones Unidas para el Desarrollo. 2005: 157-231.

Milosavljevic, Vivian. "Estadísticas para la equidad de género: magnitudes $y$ tendencias en América Latina". Cuadernos de la CEPAL 92. Santiago de Chile: CEPAL, UNIFEM, 2007.

Molyneux, Maxine. "Change and Continuity in Social Protection in Latin America: Mothers at the Service of the State?". Documento número 1. Geneva: UNRISD, 2007.

Molyneux, Maxine. "Mothers at the Service of the New Poverty Agenda: Progresa/Oportunidades, Mexico's Conditional Transfer Programme". Social Policy \& Administration 40 (4). Wiley-Blackwell, 2006: 425-449.

Molyneux, Maxine. "Más allá del debate sobre el trabajo doméstico". Rodríguez, Dinah y Jennifer Cooper. El debate sobre el trabajo doméstico. México: UnAM, 2005.

Molyneux, Maxine. Women's Movements in International Perspective: Latin America 
and Beyond. Basingstoke. Palgrave $y$ London. Institute of Latin American Studies. University of London. 2001.

O'Connor, Julia; Ann Shola Orloff y Sheila Shaver. States, Markets, Families. Gender, Liberalism and Social Policy in Australia, Canada, Great Britain and The United States. England: Cambridge University Press, 1999.

\section{OIT . Panorama Laboral 2005: América Latina} y el Caribe (Avance primer semestre). Lima: OIT. Oficina Regional para América Latina y el Caribe, 2005.

Orloff, Ann Shola. "Gender and the Welfare State". Institute for Research on Poverty Discussion Paper 1082-96. University of Wisconsin-Madison, 1996.

Palma, Julieta y Raúl Urzúa. "Políticas contra la pobreza y ciudadanía social: el caso de Chile Solidario". Colección: Políticas sociales/12. UNESCO, 2005.

Pérez Sáinz, Juan Pablo. From the Finca to the Maquila: Labour and Capitalist Development in Central America. Colorado: Boulder, Westview Press, 1999.

Rangel, Marta. "Género y trabajo en el sistema de protección Chile Solidario: la experiencia local en dos comunas de la región metropolitana". Riquelme, Verónica y María Elena Valenzuela (editoras). Chile Solidario y los desafíos de la igualdad. Proyecto género, pobreza y empleo. Oficina Internacional del Trabajo y Programa de las Naciones Unidas para el Desarrollo. 2005: 233-275.

Real Academia Española. Sitio Oficial del Diccionario de la Real Academia Española. 2008. [Definición en línea] En: <http://buscon.rae. es/draeI/Srvlt Consulta? TIPO_ $\mathrm{BUS}=3 \& \mathrm{LEMA}=\mathrm{p}$ at $\mathrm{ri}$ a r c a d o > [consultado octubre, 2008].
Riquelme, Verónica y Valenzuela, María Elena (editoras). Chile Solidario y los desafíos de la igualdad. Proyecto género, pobreza $y$ empleo. Oficina Internacional del Trabajo y Programa de las Naciones Unidas para el Desarrollo, 2005.

Ruz, Miguel Ángel y Julieta Palma. Análisis del proceso de elaboración e implementación del sistema Chile Solidario. Instituto de asuntos públicos, Departamento de políticas públicas, Universidad de Chile. 2005. [Artículo en línea] En: $<$ http://www.ciudadania.uchile.cl/docs/ informechilesolidario.pdf $>$ [consultado octubre, 2008].

Saca, Antonio. Casa Presidencial Gobierno de El Salvador. 2005. [Artículo en línea] En: <http://www.casapres.gob.sv/presidente/ discursos/2005/12/disc2201.html> [consultado varias veces entre julio $y$ setiembre, 2008].

Sagot, Montserrat. "Comentario al libro Domesticar el bienestar en América Latina”. Mesa redonda. 19 de junio. Ciudad Universitaria Rodrigo Facio. Universidad de Costa Rica. 2008.

Sainsbury, Diane. Gender and Welfare State Regimes. Oxford: Oxford University Press, 1999.

Sainsbury, Diane. "Introduction". Sainsbury, Diane (editor). Gender, Equality and Welfare States. United States. Cambridge University Press, 1996: 1-11.

Schkolnik, Mariana. "Tensión entre familia y trabajo". Arriagada, Irma y Verónica Aranda (editoras). Cambio de las familias en el marco de las transformaciones globales: necesidad de políticas públicas eficaces. Santiago. CEPAL/FNUAP, 2004: 97-118.

Secretaría Técnica del Programa avancemos. AVANCEMOS-Programa de Transferencias 
Monetarias Condicionadas. Costa Rica Rectoría del Sector Social y Lucha contra la Pobreza. Viceministerio de Desarrollo Social. 2008. [Artículo en línea] En: $<$ http://www2.ohchr.org/english/issues/ poverty/expert/docs/responses/Costa $\% 20$ Rica.pdf > [consultado julio, 2009].

Serrano, Claudia. "Claves de la política social para la pobreza". Manuscrito no publicado. 2005a.

Serrano, Claudia. "Familia como unidad de intervención de políticas sociales. Notas sobre el Programa Puente-Chile Solidario". Politica hacia las familias, protección e inclusión sociales. Santiago. CEPAL, 2005b: 14.

Serrano, Claudia. La politica social de la sociedad global en América Latina, Nuevos programas de protección social. Santiago: CEPAL, 2005c.

Serrano, Claudia. "La politica social en la globalización. Programas de protección en América Latina". Serie mujer y desarrollo. $2005 \mathrm{~d}: 78$.

Sojo, Carlos y Pérez Sáinz, Juan Pablo. "Reinventar lo social en América Latina". Sojo, Carlos (editor). Desarrollo social en América Latina: temas y desafíos en América Latina. San José: Flacso, 2002.

Texler, Marcia. "Review on Theorizing Patriarchy by Sylvia Walby". Contemporary Sociology 20 (3), mayo. American Sociological Association, 1991: 351-352.

Therborn, Göran. Between Sex and Power, Family in the World, 1900-2000. England: Routledge, 2004.

Valencia, Enrique. "Las transferencias monetarias condicionadas como política social en América Latina. Un balance: aportes, límites y debates". The Annual Review of Sociology 34. Annual Review. 2008: 499-524.

Veras, Fabio y Britto, Tatiana. "Encarnando las limitaciones en la capacidad para transferencias monetarias condicionadas en Latinoamérica: Los casos de El Salvador y Paraguay". Internacional Poverty Centre 38. Programa Red Solidaria, 2008. [Revista en línea] En: <http://www.redsolidaria.gob.sv/ component/option,com_docman/task,cat_ view/gid,42/> [consultado agosto, 2008].

Villatoro, Pablo. "Las transferencias condicionadas en América Latina: Luces y sombras". Documento de la CEPAL para el Seminario Internacional: Evolución y desafíos de los programas de transferencias condicionadas. Brasil. 20 y 21 de noviembre de 2007. [Artículo en línea] En: <http://www.cepal.org/ dds/aeci/programas.htm $>$ [consultado noviembre, 2008].

Walby, Sylvia. Gender Transformations. London and New York: Routledge, 1997.

Walby, Silvia. "The European Union and Gender Equality: Emergent Varieties of Gender Regime”. Social Politics 11 (1). Oxford University, 2004: 4-29.

\section{ENTREVISTAS}

Ávalos, Marta Carolina. Directora técnica Programa Red Solidaria. Entrevista 04 de marzo, Juliana Martínez. El Salvador. 2008.

Carrera, Carola. Investigadora de HUMANAS. Entrevista 06 de junio, Koen Voorend y Ariana Araujo. Santiago de Chile. 2008.

Raczysnki, Dagmar. Socióloga de Asesorías para el Desarrollo. Entrevista 04 de junio, Koen Voorend y Ariana Araujo. Santiago de Chile. 2008. 
